\title{
Low-Delay Joint Source-Channel Mappings for the Gaussian MAC
}

\author{
Johannes Kron, Fady Alajaji, and Mikael Skoglund
}

\begin{abstract}
The bivariate Gaussian multiterminal source coding problem with transmission over the Gaussian multiple-access channel is studied. We propose the use of low-delay joint sourcechannel mappings and show how performance saturation, which is unavoidable with linear transmission, can be overcome by optimizing the mappings. The optimized mappings are in general nonlinear and perform a combination of hard and soft decision signaling for the error-resilient transmission of analog data.
\end{abstract}

Index Terms-Joint source-channel coding, low-delay transmission, multi-terminal source coding, Gaussian multiple-access channel, correlated sources, mean square error.

\section{INTRODUCTION}

W E study the bivariate Gaussian multiterminal source coding problem with transmission over the Gaussian multiple-access channel (MAC). This problem differs from the chief executive officer (CEO) problem [1], [2] in that, here, the source variable at each terminal should be estimated; in contrast to the CEO problem where the terminals have access to noisy observations of a single underlying source variable that should be estimated.

For the CEO problem and the Gaussian MAC, it is known that uncoded, or linear, transmission is optimal [3]. However, in the multiterminal source coding problem, it has been shown that linear transmission is optimal only for signal-to-noise ratios (SNRs) below a certain threshold [4]. Motivated by lowdelay constraints, in for example sensor networks or closedloop control applications, we look at analog joint sourcechannel mappings. There are several practical examples of point-to-point communication with this kind of analog sourcechannel mappings [5]-[12]. There are not as many results for multi-user scenarios as considered in this paper; see [13]-[17] for a few of the existing multi-user results.

In what follows, we explicitly design optimized, low-delay transmission schemes for the Gaussian multiterminal source coding problem and the Gaussian MAC. This problem was also considered recently in [17], however using parametrized mappings, and in [13], [16], assuming orthogonal channels. The novel contribution of this paper is to consider numerically optimized mappings for the interfering Gaussian MAC. We show that the optimized mappings are in general nonlinear, performing a combination of hard and soft decision signaling for the error-resilient transmission of analog data.

Manuscript received September 12, 2013. The associate editor coordinating the review of this letter and approving it for publication was R. Souza.

This work was supported in part by the Swedish governmental agency for innovation systems (VINNOVA), the Swedish Research Council (VR), and the Natural Sciences and Engineering Research Council (NSERC) of Canada.

J. Kron (Fr. J. Karlsson) and M. Skoglund are with the School of Electrical Engineering, Royal Institute of Technology (KTH), Sweden (e-mail: \{johk, skoglund\}@ee.kth.se).

F. Alajaji is with the Department of Mathematics and Statistics, Queen's University, Canada (e-mail: fady@mast.queensu.ca).

Digital Object Identifier 10.1109/LCOMM.2013.120413.132088

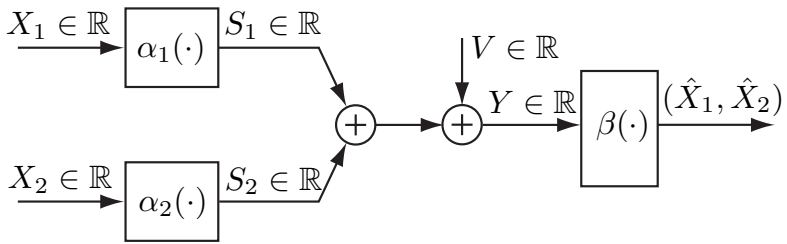

Fig. 1. Overview of the joint source-channel coding scheme for the Gaussian MAC with correlated sources. $\left(\hat{X}_{1}, \hat{X}_{2},\right)=\beta(Y)=\left(\beta_{1}(Y), \beta_{2}(Y)\right)$.

\section{Problem Formulation}

We consider a scenario where two spatially separated sensor nodes, each measure a Gaussian random variable $X_{i}$, which is to be transmitted to a joint receiver. The Gaussian random variables, $X_{1}$ and $X_{2}$, are identically distributed and have zero mean and variance $\sigma_{X}^{2}$. Furthermore, they are correlated with a correlation coefficient defined by $\rho \triangleq E\left[X_{1} X_{2}\right] / \sigma_{X}^{2}$.

Due to low-delay constraints, we consider transmission on a sample-by-sample basis where sensor $i$ maps its measurement directly to the channel space by the mapping $\alpha_{i}$ and transmits $S_{i}=\alpha_{i}\left(X_{i}\right) \in \mathbb{R}$. The transmissions are made over the Gaussian MAC and the received signal is given by

$$
Y=\alpha_{1}\left(X_{1}\right)+\alpha_{2}\left(X_{2}\right)+V,
$$

as shown in Fig. 1, where $V$ is additive white Gaussian noise, independent of $X_{1}$ and $X_{2}$, with variance $\sigma_{V}^{2}$. At the receiver, the source variables $\left(X_{1}, X_{2}\right)$ are reconstructed by the mapping $\hat{X}_{i}=\beta_{i}(Y), i=1,2$. The overall objective is to find the combination of $\left(\alpha_{1}, \alpha_{2}, \beta_{1}, \beta_{2}\right)$ that minimizes the average mean squared error, defined according to

$$
\mathrm{MSE} \triangleq \frac{1}{2} \sum_{i=1}^{2} E\left[\left(X_{i}-\hat{X}_{i}\right)^{2}\right] .
$$

The minimization should be done under the following transmission power constraint: ${ }^{1}$

$$
\frac{1}{2} \sum_{i=1}^{2} E\left[\left\|\alpha_{i}\left(X_{i}\right)\right\|^{2}\right] \leq P .
$$

\section{ANALYSIS}

Using the Lagrange multiplier method [18], we turn the constrained optimization problem of minimizing (2) subject to (3) into an unconstrained problem by first forming the Lagrange cost function

$$
J\left(\alpha_{1}, \alpha_{2}, \beta_{1}, \beta_{2}\right)=\sum_{i=1}^{2}\left\{\frac{1}{2} E\left[\left(X_{i}-\hat{X}_{i}\right)^{2}\right]+\lambda E\left[\left\|\alpha_{i}\left(X_{i}\right)\right\|^{2}\right]\right\}
$$

${ }^{1}$ It is possible to have individual power constraints on each transmitter; the only minor modification that needs to be made in the analysis is to have individual Lagrange multipliers (i.e., $\lambda_{1}$ and $\lambda_{2}$ instead of $\lambda$ ). Note that individual power constraints are more restrictive and hence give higher MSE. 
and next expressing the problem as

$$
\min _{\alpha_{1}, \alpha_{2}, \beta_{1}, \beta_{2}} J\left(\alpha_{1}, \alpha_{2}, \beta_{1}, \beta_{2}\right) .
$$

Here $\lambda \geq 0$ is a Lagrange multiplier that is used to control the average power. If for a given $\lambda$, we solve the unconstrained problem in (5) and find that the power constraint in (3) is fulfilled with equality, the solution we have obtained is also a solution to the constrained optimization problem [18].

The minimization problem in (5) is still very hard to solve due to interdependencies between the components that are optimized and since the problem is nonconvex. To get around these difficulties we proceed as in vector quantization (VQ) optimization [19], [20] and state necessary conditions for the optimality of each component. Based on these necessary conditions, we optimize the system iteratively, one component at a time while keeping the other components fixed.

\section{A. Necessary Conditions for Optimality}

Beginning with $\alpha_{1}$, if we assume that $\left(\alpha_{2}, \beta_{1}, \beta_{2}\right)$ are fixed, the optimal $\alpha_{1}$ is given by

$$
\begin{aligned}
\alpha_{1} & =\arg \min _{\alpha_{1}} J\left(\alpha_{1}, \alpha_{2}, \beta_{1}, \beta_{2}\right) \\
& =\arg \min _{\alpha_{1}}\{\sum_{i=1}^{2}(\frac{1}{2} \underbrace{E\left[\left(X_{i}-\hat{X}_{i}\right)^{2}\right]}_{\mathrm{MSE}_{i}})+\lambda \underbrace{E\left[\left\|\alpha_{1}\left(X_{1}\right)\right\|^{2}\right]}_{P_{1}}\} .
\end{aligned}
$$

Using Bayes' rule, the MSE of user $i$ can be expressed as

$$
\begin{aligned}
\mathrm{MSE}_{i} & =\int p\left(x_{1}\right) \int p\left(x_{2} \mid x_{1}\right) \times \\
& \int p\left(y \mid \alpha_{1}\left(x_{1}\right), \alpha_{2}\left(x_{2}\right)\right)\left(x_{i}-\beta_{i}(y)\right)^{2} \mathrm{~d} y \mathrm{~d} x_{2} \mathrm{~d} x_{1},
\end{aligned}
$$

where $p(\cdot)$ and $p(\cdot \mid \cdot)$ denote probability densities and conditional densities, respectively. The average power of user $i$ is given by

$$
P_{i}=\int p\left(x_{i}\right)\left\|\alpha_{i}\left(x_{i}\right)\right\|^{2} \mathrm{~d} x_{i} .
$$

Looking at (7) and (8), we can see that the objective function in (6) can be expressed as an integration over $x_{1}$ with an integrand of the form $p\left(x_{1}\right) f\left(x_{1}\right)$. Since $p\left(x_{1}\right)$ by definition is nonnegative for all $x_{1}$, we can find the optimal $\alpha_{1}$ by minimizing $f\left(x_{1}\right)$ for each value of $x_{1}$ in the following way:

$$
\begin{aligned}
\alpha_{1}\left(x_{1}\right) & =\arg \min _{s_{1} \in \mathbb{R}}\left\{\int p\left(x_{2} \mid x_{1}\right) \int p\left(y \mid s_{1}, \alpha_{2}\left(x_{2}\right)\right) \times\right. \\
& \left.\frac{1}{2}\left(\left(x_{1}-\beta_{1}(y)\right)^{2}+\left(x_{2}-\beta_{2}(y)\right)^{2}\right) \mathrm{d} y \mathrm{~d} x_{2}+\lambda\left\|s_{1}\right\|^{2}\right\} .
\end{aligned}
$$

This equation is a necessary condition for $\alpha_{1}$ to be part of the optimal solution and if $\left(\alpha_{2}, \beta_{1}, \beta_{2}\right)$ are given beforehand it provides the optimal mapping $\alpha_{1}$. In a similar way, $\alpha_{2}$ can be found by assuming that $\left(\alpha_{1}, \beta_{1}, \beta_{2}\right)$ are fixed.

If we move on to the receiving side and assume that $\alpha_{1}$ and $\alpha_{2}$ are given, we can find the optimal estimators. Since we are using the MSE as our cost function, $\beta_{i}$ is given by the conditional expected mean

$$
\hat{x}_{i}=\beta_{i}(y)=E\left[X_{i} \mid y\right] .
$$

\section{B. Design Algorithm}

Based on the necessary conditions for optimality, we now propose a design algorithm that iterates between optimizing the mappings at the sensor nodes and the receiver. This kind of iterative optimization does not in general guarantee convergence to the global optimum. Nevertheless, it has successfully been used in many applications such as VQ design [19], [20] and design of joint source-channel mappings for different scenarios, see for example [6], [15], [21]. For a fixed SNR $\triangleq P / \sigma_{V}^{2}$ and source correlation $\rho$, the design procedure is stated in Algorithm 1.

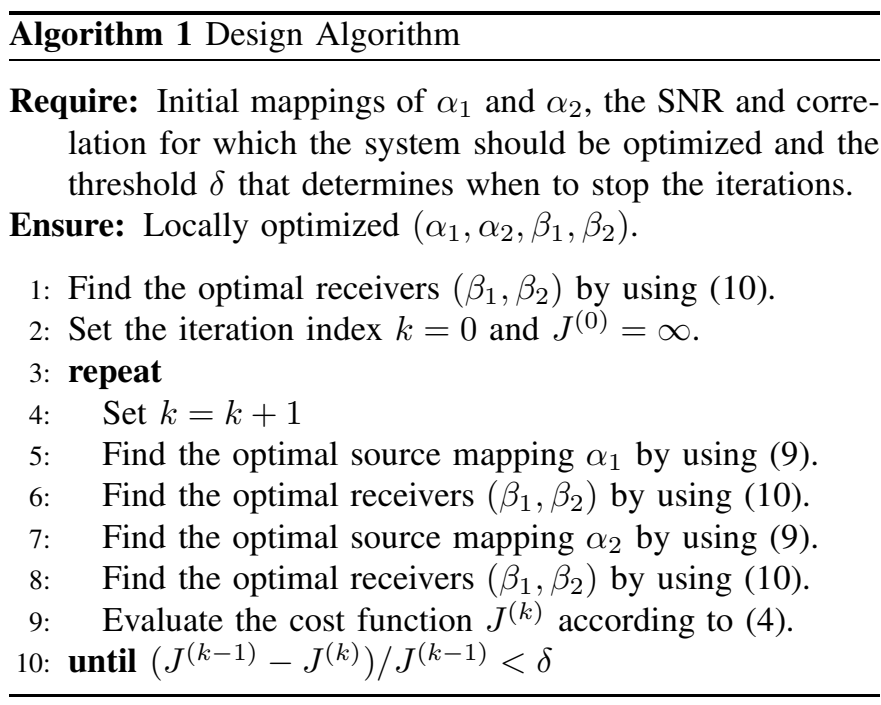

In our simulations we have used $\delta=10^{-4}$ to determine when to stop the iteration. It is clear that the algorithm will converge since the cost function is bounded from below and reduced by each iteration. We use a combination of two methods to avoid poor local minimums. We first choose a good initialization in the first step of the design algorithm, see Section VI-A. We also use a post-processing step similar to noisy channel relaxation [22]. Assume that we have designed systems for a range of SNR points, for example $\{10,15,20,25\} \mathrm{dB}$. We now initialize the algorithm with the system designed for the smallest SNR and optimize for the second smallest SNR. If the new system performs better than the system that was previously optimized for this SNR point we keep it. This process is repeated for each SNR point. Once we reach the largest SNR point we repeat the process backwards until we reach the smallest SNR. By stepping back and forward a couple of times, we can avoid that a system designed for a particular SNR performs much worse than any other system. This process was also used in [21].

\section{LineAR TRANSMission AND Distortion BOUND}

The distortion region of this problem is in general unknown. Since the sources are correlated and the communication is over the Gaussian MAC, the source-channel separation theorem does not hold. For the purpose of evaluating the performance of our optimized solutions we compare the results to linear transmission and the distortion lower bound from [4]. The lower bound is tight for $\mathrm{SNR}=P / \sigma_{V}^{2} \leq \rho /\left(1-\rho^{2}\right)$ in which 
case linear transmission is optimal. In [4], a joint sourcechannel code is constructed that performs close to the lower bound for high SNRs. However, both the lower bound and the joint source-channel code rely on infinite block lengths.

\section{IMPLEMENTATION ASPECTS}

For the actual implementation of the formulas in (9) and (10), we need to make some modifications and approximations. First, we restrict the output of $\alpha_{i}$ to a finite set of modulation points $\mathcal{S}$ given by

$$
\mathcal{S}=\left\{-\Delta \frac{M-1}{2},-\Delta \frac{M-3}{2}, \ldots, \Delta \frac{M-3}{2}, \Delta \frac{M-1}{2}\right\},
$$

where $\Delta$ determines the resolution of the modulation points and $M$ is the number of points in $\mathcal{S}$. As $M$ and $\Delta$ approach infinity and zero, respectively, we have analog modulation. ${ }^{2}$ We have kept $\Delta(M-1) / 2=4$ and used an $M$ in the range $[321,1281]$ depending on the SNR. The $M$ we have chosen is such that there is no significant improvement in using a larger value. The minimization in (9) is thus made over $s_{1} \in \mathcal{S}$. Similarly, the received signal $y$ is pre-quantized by a nearest neighbor quantizer to a finite set $\mathcal{Y}=\{y: y=$ $\left.s_{1}+s_{2}, \forall s_{1}, s_{2} \in \mathcal{S}\right\}$. The receiver needs thus only to be defined for a finite input set. Our final modification is to use Monte-Carlo samples for $\left(X_{1}, X_{2}\right)$; this allows us to write discretized versions of (9) and (10). The value of the Lagrange multiplier $\lambda$ depends on the scenario; typical values that we have used range from $4 \cdot 10^{-6}$ to 0.1 . In general, $\lambda$ should be decreased (increased) if the SNR or correlation is increased (decreased). Better control of the final power consumption can be achieved by increasing or decreasing $\lambda$ in each iteration if the total power is too low or too high, respectively. This is particularly useful, if (as in this paper) the modulation points are finite, $P$ is fixed, and $\sigma_{V}^{2}$ is changed to give certain SNR.

\section{Vi. Performance Evaluation}

\section{A. Initialization}

After running the design algorithm a couple of times, it became clear that staircase functions are good starting points for the iterative design procedure. We therefore initialize $\alpha_{1}$ with a staircase function with $L$ steps. For $\left|x_{1}\right| \leq 4$, this can be expressed as

$$
\alpha_{1}\left(x_{1}\right)=\left\lfloor Q_{L}\left(x_{1}\right) \frac{2}{L-1} \max _{s_{1} \in \mathcal{S}} s_{1}\right\rceil_{\mathcal{S}}
$$

where

$$
\begin{aligned}
Q_{L}\left(x_{1}\right) & =\left\lfloor\frac{x_{1}}{4} \frac{L-1}{2}-\frac{\operatorname{even}(L)}{2}\right\rceil_{\mathbb{Z}}+\frac{\operatorname{even}(L)}{2}, \\
\operatorname{even}(L) & = \begin{cases}1 & \text { if } L \text { is even } \\
0 & \text { otherwise, }\end{cases}
\end{aligned}
$$

and $\lfloor\cdot\rangle_{\mathcal{S}}$ returns the closest point (in terms of Euclidean distance) in the set $\mathcal{S}$. The best choice of $L$ typically depends on both the correlation and the SNR. In our simulations we have used $L$ in the range from 2 to 20 . The mapping $\alpha_{2}$ has been initialized identical to zero for all inputs.

\footnotetext{
${ }^{2}$ Of course one has to make sure that $1 / \Delta$ does not grow faster than $M$ in which case the product $\Delta M \rightarrow 0$.
}

\section{B. Numerical Results}

In Fig. 2, we plot the results for two different source correlations, namely, $\rho=0.5$ and $\rho=0.9$. The results are plotted as $\mathrm{SDR} \triangleq 10 \log _{10} \sigma_{X}^{2} / \mathrm{MSE}$ versus SNR. The curves show practical systems where the encoders have been optimized for certain SNR points that are marked by circles in the figures. We assume that the true SNR is known by the receiver and that the decoder is updated accordingly.

We can see that the optimized systems perform very well and overcome the saturation that is unavoidable with linear transmission. The systems are robust against SNR mismatch on the transmitting side. The system performs about $0.6-$ $1 \mathrm{~dB}$ better than the scalar quantizer linear coder (SQLC) constructed in [17] for $\rho=0.5$. When looking at $\rho=0.9$ the gain is about the same for low SNR but only slightly better at high SNR where in fact the optimized encoders are similar to SQLC. As predicted by the theoretical results in Section IV, linear transmission works well in the low-SNR region. In a real system where there are time variations, a feedback link could be utilized to ensure that the best performing encoders are always used. Since we are plotting the results as SDR, the distortion lower bound mentioned in Section IV become an SDR upper bound. It should be emphasized that the upper bound is an asymptotic result in the sense of infinite block lengths of the source samples as well as in the channel coding part. The gap between the upper bound and our low-delay system is therefore not surprising.

\section{Encoders and Decoders}

We shall now take a closer look at the encoder-decoder structure. In Fig. 3, we show encoders (left) and decoders (right) for different correlations and SNRs. The interaction between the two encoders is easiest understood by looking at the corresponding joint decoders to the right in Fig. 3 . The staircase-like encoder mappings perform a combination of hard and soft decision signaling, which makes the reconstruction points fill the source space in an efficient way. This is the reason why the optimized mappings perform better than linear transmission where the decoder would be the straight line $\hat{X}_{2} \equiv \hat{X}_{1}$ regardless of the SNR and correlation. The power allocated to the second user is in general decreased as the SNR is increased and increased as the correlation is increased; for example, given that $\rho=0.9,\left(P_{1}, P_{2}\right)=(1.54 P, 0.46 P)$ at an SNR of $20 \mathrm{~dB}$ and $\left(P_{1}, P_{2}\right)=(1.94 P, 0.06 P)$ at an SNR of $30 \mathrm{~dB}$. An overall equal power allocation can be achieved by means of time sharing.

\section{CONCLUSIONS}

We have proposed the use of optimized source-channel mappings for the bivariate Gaussian multiterminal source coding problem with transmission over the Gaussian MAC. The optimized nonlinear mappings fill a gap between linear transmission and existing upper bounds. The main advantage of the optimized mappings is their low-delay properties, due to their operation on a sample-by-samples basis. Although we focused on the matched bandwidth system (where one source symbol is sent per channel use), similar results can be obtained for the unequal bandwidth case. 


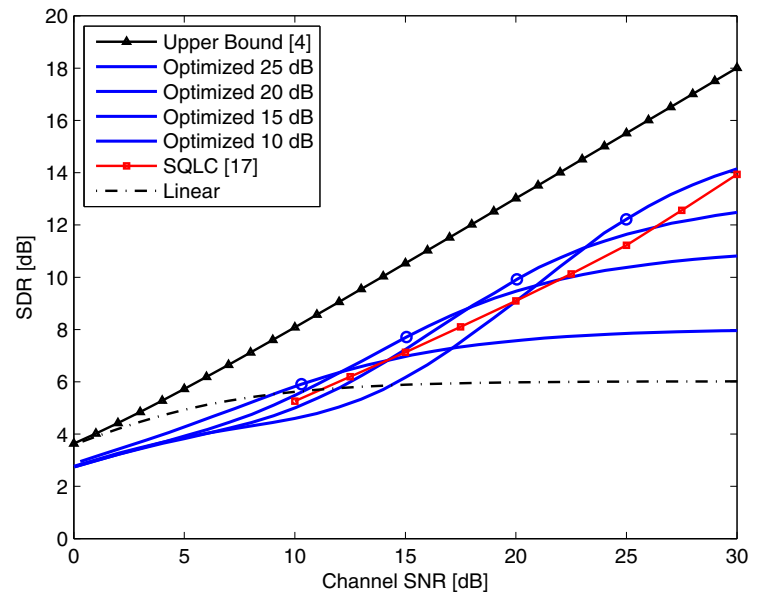

(a) Source correlation $\rho=0.5$

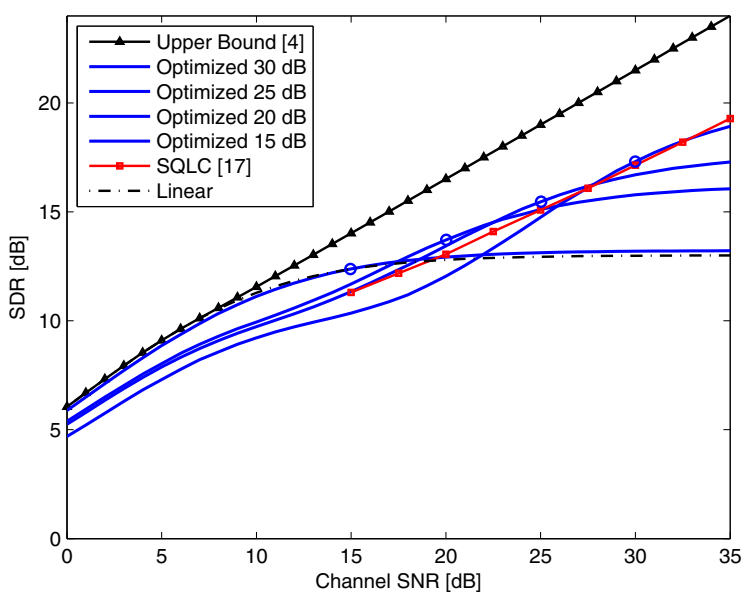

(b) Source correlation $\rho=0.9$

Fig. 2. Simulation results. Circles mark the points where the mappings are optimized. The results of [17] was kindly provided by P.A. Floor.
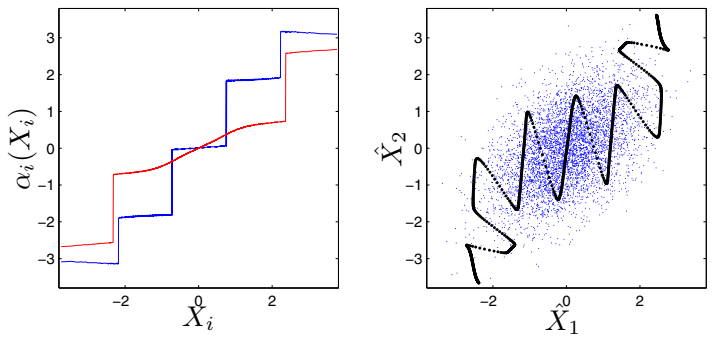

(a) $\mathrm{SNR}=15 \mathrm{~dB}, \rho=0.5 . P_{1}=1.7 P, P_{2}=0.3 P$.
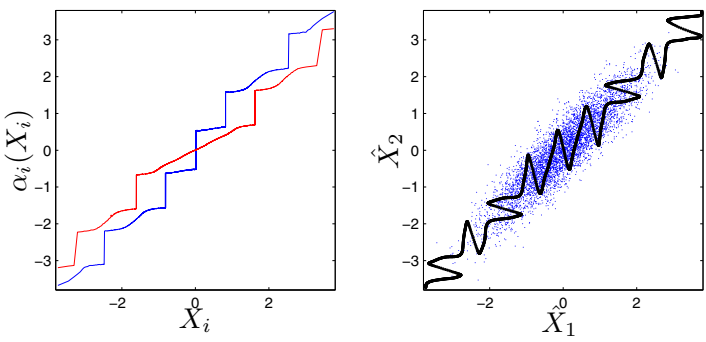

(b) $\mathrm{SNR}=20 \mathrm{~dB}, \rho=0.9 . P_{1}=1.5 P, P_{2}=0.5 P$.

Fig. 3. Encoders (left) and their corresponding decoders (right) optimized for different SNRs and correlations. In the figures to the right, the dotted lines show reconstruction points $\left(\hat{x}_{1}, \hat{x}_{2}\right)$ and the small dots are samples from the distribution of $\left(X_{1}, X_{2}\right)$.

\section{REFERENCES}

[1] T. Berger, Z. Zhang, and H. Viswanathan, "The CEO problem [multiterminal source coding]," IEEE Trans. Inf. Theory, vol. 42, no. 3, pp. 887-902, May 1996.

[2] H. Viswanathan and T. Berger, "The quadratic Gaussian CEO problem," IEEE Trans. Inf. Theory, vol. 43, no. 5, pp. 1549-1559, 1997.

[3] M. Gastpar, "Uncoded transmission is exactly optimal for a simple Gaussian "sensor" network," IEEE Trans. Inf. Theory, vol. 54, no. 11, pp. 5247-5251, Nov. 2008.

[4] A. Lapidoth and S. Tinguely, "Sending a bivariate Gaussian over a Gaussian MAC," IEEE Trans. Inf. Theory, vol. 56, no. 6, pp. 27142752, 2010.

[5] V. Vaishampayan, "Combined source-channel coding for bandlimited waveform channels," Ph.D. dissertation, University of Maryland, 1989

[6] A. Fuldseth and T. A. Ramstad, "Bandwidth compression for continuous amplitude channels based on vector approximation to a continuous subset of the source signal space," in Proc. 1997 International Conference on Acoustics, Speech and Signal Processing, pp. 3093-3096.

[7] B. Chen and G. W. Wornell, "Analog error-correcting codes based on chaotic dynamical systems," IEEE Trans. Commun., vol. 46, no. 7, pp. 881-890, July 1998.

[8] S. Chung, "On the construction of some capacity-approaching coding schemes," Ph.D. dissertation, MIT, 2000.

[9] V. Vaishampayan and S. I. R. Costa, "Curves on a sphere, shift-map dynamics, and error control for continuous alphabet sources," IEEE Trans. Inf. Theory, vol. 49, no. 7, pp. 1658-1672, July 2003.

[10] N. Wernersson, M. Skoglund, and T. Ramstad, "Polynomial based analog source-channel codes," IEEE Trans. Commun., vol. 57, no. 9, pp. 2600-2606, Sept. 2009.

[11] F. Hekland, P. A. Floor, and T. A. Ramstad, "Shannon-Kotel'nikov mappings in joint source-channel coding," IEEE Trans. Commun., vol. 57, no. 1, pp. 94-105, Jan. 2009.
[12] A. A. Saleh, F. Alajaji, and W.-Y. Chan, "Power-constrained bandwidthreduction source-channel mappings for fading channels," in Proc. 2012 Biennial Symp. Commun., pp. 85-90.

[13] N. Wernersson, J. Karlsson, and M. Skoglund, "Distributed quantization over noisy channels," IEEE Trans. Commun., vol. 57, no. 6, pp. $1693-$ 1700 , June 2009.

[14] N. Wernersson and M. Skoglund, "Nonlinear coding and estimation for correlated data in wireless sensor networks," IEEE Trans. Commun., vol. 57, no. 10, pp. 2932-2939, Oct. 2009.

[15] J. Karlsson and M. Skoglund, "Optimized low-delay source-channelrelay mappings," IEEE Trans. Commun., no. 5, pp. 1397-1404, May 2010.

[16] E. Akyol, K. Rose, and T. Ramstad, "Optimized analog mappings for distributed source-channel coding," in 2010 Proc. IEEE Data Compression Conference, pp. 159-168.

[17] P. A. Floor, A. N. Kim, N. Wernersson, T. A. Ramstad, M. Skoglund, and I. Balasingham, "Zero-delay joint source-channel coding for a bivariate Gaussian on a Gaussian MAC," IEEE Trans. Commun., vol. 60, no. 10, pp. 3091-3102, Oct. 2012.

[18] H. Everett III, "Generalized Lagrange multiplier method for solving problems of optimum allocation of resources," Operations Research, vol. 11, no. 3, pp. 399-417, 1963.

[19] S. P. Lloyd, "Least squares quantization in PCM," IEEE Trans. Inf. Theory, vol. 28, no. 2, pp. 129-137, Mar. 1982.

[20] Y. Linde, A. Buzo, and R. M. Gray, "An algorithm for vector quantizer design," IEEE Trans. Commun., vol. 28, no. 1, pp. 84-95, Jan. 1980.

[21] N. Farvardin and V. Vaishampayan, "Optimal quantizer design for noisy channels: an approach to combined source-channel coding," IEEE Trans. Inf. Theory, vol. 33, no. 6, pp. 827-838, Nov. 1987.

[22] S. Gadkari and K. Rose, "Noisy channel relaxation for VQ design," in Proc. 1996 International Conference on Acoustics, Speech and Signal Processing, pp. 2048-2051. 